\title{
APPEARANCE AND DISTRIBUTION OF NEURONAL CELL SURFACE AND SYNAPTIC VESICLE ANTIGENS IN THE DEVELOPING RAT SUPERIOR CERVICAL GANGLION ${ }^{1}$
}

\author{
KAREN F. GREIF' ${ }^{2}$ AND LOUIS F. REICHARDT \\ Department of Physiology, School of Medicine, University of California, San Francisco, California 94143
}

Received December 11, 1981; Revised February 17, 1982; Accepted February 22, 1982

\begin{abstract}
Monoclonal antibodies directed against a neuronal cell surface heparan sulfate proteoglycan and against a synaptic vesicle protein were used to study the postnatal development of ganglionic neurons and synapses in the rat superior cervical ganglion. Antigen levels in developing ganglia were quantitated by radioimmune assays. Localization of antigens in adult and developing ganglia was carried out using peroxidase-antiperoxidase immunocytochemistry at the light microscopic level. Ultrastructural staining patterns in adult ganglia also were studied.

The time course of antigen increases parallels those in previous reports on the accumulation of neurotransmitter enzymes within the ganglion. Both synaptic and surface antigens increase postnatally, with the most rapid changes occurring during the 2nd week. Antibodies stain adult tissue in patterns consistent with the expected distribution of antigens: antibodies directed against synaptic vesicles stain synaptic terminals and cell cytoplasm and those directed against surface proteoglycan stain the plasma membranes of neuronal cell bodies and processes. Variable staining of the cell cytoplasm also is observed. No apparent changes in antigen distribution are observed with the light microscope during development. Variations in the time course of the development of antigens associated with different portions of the proteoglycan molecule suggest that the intracellular processing of this molecule may vary during development.
\end{abstract}

The mammalian superior cervical ganglion (SCG) has been a popular model system for the study of neuronal and synaptic development (see Black, 1978, for review). It is accessible and easy to manipulate and has a relatively simple organization (Gabella, 1976; Eranko, 1972). The principal ganglionic neurons, which receive cholinergic input from the spinal cord, synthesize norepinephrine and adrenergically innervate a number of peripheral targets, most notably the iris and salivary glands. The ganglion also contains small clusters of catecholaminergic "small intensely fluorescent" (SIF) cells of possible neu-

\footnotetext{
'We thank Frank Novak for assistance with thin sectioning, Eric Outwater for preparation of the IgG-peroxidase conjugate, and Drs. 7ach Hall and Jeffrey Browning for helpful criticisms of the manuscript. This research was supported by grants to L. F. R. from the National Science Foundation, Muscular Dystrophy Association, McKnight Foundation, March of Dimes Birth Defects Foundation, and Wills Foundation. K. F. G. was supported by a Muscular Dystrophy Association Postdoctoral Fellowship and National Institutes of Health Training Grant 2-404945-24171-3. L. F. R. is a Sloan Foundation Fellow.

${ }^{2}$ To whom correspondence should be addressed at her present address: Department of Biology, Bryn Mawr College, Bryn Mawr, PA 19010 .
}

rohumoral function (Eranko and Eranko, 1971) and supporting cells of glial origin. Most synapses within the SCG are cholinergic, with preganglionic input arising from the cervical spinal cord. Recent evidence (Kondo et al., 1980) suggests that there is also some synaptic contact between the adrenergic neurons of the ganglion.

Neuronal maturation and the progress of synapse formation have been studied by electron microscopy (Eranko, 1972; Black et al., 1971; Smolen and Raisman, 1980) and by assay of neurotransmitter synthetic enzymes. The principal synthetic enzyme for acetylcholine, choline acetyltransferase (CAT), is localized in presynaptic terminals within the SCG and overall levels of CAT within the ganglion have been used to estimate the progress of synapse formation (Black et al., 1971, 1972; Black and Geen, 1973). Tyrosine hydroxylase (TH), the rate-limiting enzyme for catecholamine synthesis, is concentrated in the cell bodies of ganglionic neurons (Black et al., 1971) and has been used to monitor neuronal maturation.

In the mouse, an identifiable SCG is present from embryonic days 13 to 15 following migration of sympathoblasts from the neural crest. Presumptive ganglionic neurons already contain catecholamines at this stage as 
measured by histofluorescence (Gabella, 1976). From the time of cell migration until birth, neuroblasts increase in size with a concomitant 40 -fold increase in $\mathrm{TH}$ levels. The increase is due to an accumulation of enzyme and not a decrease in the rate of enzyme degradation (Coughlin et al., 1978). Axons of ganglionic neurons reach their targets shortly before birth, with further elaboration of synapses occurring postnatally. After birth, ganglionic neurons undergo final maturation with an increase in cell diameter, an expansion of dendritic territory, and a 5- to 10 -fold increase in TH levels. Neurons cease mitotic activity between days 7 and 9 postnatal (Black et al., 1971; Eranko, 1972). Glial proliferation occurs largely postnatally and continues for several weeks.

Recognizable synapses within the SCG are rare at birth; estimates range from 1 to $10 \%$ of adult values (Black et al., 1971; Smolen and Raisman, 1980). Synaptogenesis takes place most rapidly during the first 2 weeks after birth as estimated by both CAT assay (Black et al., 1971, 1972) and by electron microscopy (Black et al., 1971; Smolen and Raisman, 1980).

The manipulation of presynaptic input has trans-synaptic effects on neuronal maturation and synaptogenesis (Black, 1978; Black et al., 1971, 1972, 1979; Black and Geen, 1973; Smolen and Raisman, 1980). Isolation of the SCG from presynaptic input at birth causes a reduction in neuronal cell division and growth and blocks the increase in TH levels. The effect of denervation is mimicked by treatment with the ganglionic blockers, chlorisondamine and pempidine (Black and Geen, 1973). This suggests that presynaptic input exerts its major influence via the direct action of acetylcholine on its postsynaptic receptor and the subsequent depolarization of ganglionic neurons. Additional trophic influences from the presynaptic nerve also may contribute to postsynaptic changes associated with denervation (Hendry and Hill, 1980).

Further studies of neuronal development have been hampered by the limited number of specific probes available to assess the progress of maturation. Recent interest in the roles of cell surface molecules in neuronal development has led to the application of immunological techniques. We have used monoclonal antibodies directed against a cell surface heparan sulfate proteoglycan and a synaptic vesicle protein to monitor postnatally neuronal and synaptic development in the rat SCG. In particular, we wished to investigate whether synapse formation and ganglionic neuron maturation occur concurrently or sequentially, how the time course of the development of these antigens is related to the accumulation of neurotransmitter enzymes previously reported, and whether the distribution of these antigens changes during the course of ganglion maturation. Two antibodies (PG 3 and PG 22) are directed against a cell surface proteoglycan with heparan sulfate (HeS) side chains (W. D. Matthew and L. F. Reichardt, manuscript in preparation). PG 3 is directed against a determinant associated with the heparan sulfate portion of the molecule; $\mathrm{PG} 22$ appears to bind either the core protein or a $\mathrm{NH}_{2}$-linked carbohydrate associated with it. The HeS proteoglycan defined by these antibodies is found in culture on neurons but not on fibroblasts or Schwann cells. Recent experiments indicate that the proteoglycan is part of a complex that induces neurite outgrowth by peripheral neurons in culture (A. D. Lander, R. Greenspan, W. D. Matthew, and I. F. Reichardt, unpublished observations). In this paper, these antibodies are used to follow the appearance and distribution of a particular cell surface antigen likely to have an important in vivo function during the maturation of ganglionic neurons. The other two antibodies (SV 30 and SV 48) bind a 65,000-daltun integral membrane protein which is found on the plasma membrane of synaptic vesicles of all neuronal cell types investigated (Matthew et al., 1981a, b). The protein is highly conserved across species and it has not been found in uninnervated tissue. These two antibodies are used to quantitate postnatal synapse formation in the SCG. A preliminary report of some of this research has been published (Greif et al., 1981).

\section{Materials and Methods \\ Quantitation of antigen}

The assay was designed to determine the concentration of a given antigen $(\mathrm{Ag})$ in whole tissue homogenates by the ability of homogenate dilutions to inhibit the binding of a limiting dilution of antibody $(\mathrm{Ab})$ in a solid phase radioimmune plate assay (RIA). The derived values permitted comparisons of Ag levels in different homogenates for the same antibody but did not allow the direct comparison of Ag levels between different Abs.

Ganglia were collected in normal saline on ice and stripped of connective tissue. Whole tissue homogenates were prepared using a glass-glass homogenizer (Kontes). The ganglia were homogenized in $5 \mathrm{~mm}$ Tris- $\mathrm{Cl}, \mathrm{pH} 8.1$ (lysis buffer), with phenylmethylsulfonyl fluoride added to reduce proteolysis. The homogenates were incubated for $30 \mathrm{~min}$ on ice in lysis buffer and were stored at $-20^{\circ} \mathrm{C}$ in $10 \%$ sucrose in phosphate-buffered saline (PBS).

Protein concentration was determined by Amido schwarz assay using bovine serum albumin (BSA) as the standard. The initial protein concentration for the dilution series was adjusted to 3 to $6 \mathrm{mg} / \mathrm{ml}$, depending on the amount of material available. Twelve dilutions were made to $1: 10,000$ in 5\% newborn calf serum in PBS.

Limiting dilutions of monoclonal antibody were determined by solid phase RIA (Klinman, 1972). Culture supernatants were diluted for PG 3 and PG 22 while an ascites fluid of SV 48 was employed. Equal volumes of $\mathrm{Ab}$ and diluted homogenate were incubated for $24 \mathrm{hr}$ at $4^{\circ} \mathrm{C}$. Samples containing PG 3 and PG 22 were spun for $15 \mathrm{~min}$ in a Microfuge (Beckman). It was found that samples of $\mathrm{SV} 48$ required faster spins to precipitate bound $\mathrm{Ab}$; these samples were spun at $100,000 \times \mathrm{g}$ in an Airfuge (Beckman) for 40 min. No differences were observed when other Abs were spun at higher velocities.

The amount of $\mathrm{Ab}$ remaining in the supernatant was determined by solid phase RIA. Samples of a crude lysed rat brain synaptosome preparation (Jones and Matus, 1974; through the hypotonic lysis step) were bound to the wells of a flexible plastic microtiter plate. Aliquots of $\mathrm{Ab}$ were added in duplicate and incubated overnight before the addition of ${ }^{125} \mathrm{I}-\mathrm{Fab}^{\prime 2}$ fragments of goat antimouse immunoglobulin. Microwells were counted in a Gamma counter (Beckman 4000) and the 50\% inhibition 
level $\left(\mathrm{I}^{50}\right)$ was determined graphically. The slope of the inhibition curves did not change markedly for homogenates at different stages of development, with increases occurring over approximately $1.2 \log$ units. Values were normalized to a $4 \mathrm{mg} / \mathrm{ml}$ initial dilution of $\mathrm{Ag}$. These values were converted to $\mathrm{I}^{50}$ milligram units per ganglion to correct for the large increase in size of the ganglia during postnatal development, according to the equation: $\mathrm{I}^{50} \mathrm{mg}$ units/ganglion $=\mathrm{I}^{50} \mathrm{mg}($ total protein $/$ sample $/$ total protein/adult ganglion).

\section{Immunocytochemistry}

Light microscopy. Rats were stunned by a blow to the head, the spinal cord and aorta were severed, and the SCGs were removed immediately. Individual ganglia were stripped of the connective tissue sheath and supported on small slices of diaphragm muscle placed on strips of cardboard. The tissue was frozen immediately by immersion in liquid nitrogen and either sectioned immediately or stored in liquid nitrogen.

The tissue was mounted on a cryostat chuck using Tissue-Tek (Miles) with care taken to avoid thawing. Sections 6 to $8 \mu \mathrm{m}$ thick were cut, mounted on slides, and air-dried. Sections not used immediately were stored at $-80^{\circ} \mathrm{C}$ with desiccant for up to 1 week before use.

Immunocytochemical staining was carried out using the three-step peroxidase-antiperoxidase (PAP) method of Sternberger (1979). After blocking with $3 \%$ goat serum in phosphate-buffered saline, sections were incubated with culture supernatants containing monoclonal $\mathrm{Ab}$ for 12 to $18 \mathrm{hr}$ at $4^{\circ} \mathrm{C}$. Linker $\mathrm{Ab}$, goat anti-mouse serum (Cappel), was preabsorbed with rat liver powder and used at 1:10 dilution for $2 \mathrm{hr}$ at $4^{\circ} \mathrm{C}$. Rat PAP was prepared according to the Sternberger (1979) method (40 $\mu \mathrm{g} / \mathrm{ml}$ of peroxidase) and preabsorbed with liver powder. The incubations were carried out for $1 \mathrm{hr}$ at $4^{\circ} \mathrm{C}$. Sections were stained using 3,3'-diaminobenzidine (DAB) and $0.01 \% \mathrm{H}_{2} \mathrm{O}_{2}$ in $0.05 \mathrm{M}$ Tris-Cl, $\mathrm{pH} 7.6$, for $15 \mathrm{~min}$ at room temperature. They were mounted in Elvanol (polyvinyl alcohol, Monsanto) and examined using a Zeiss photomicroscope equipped for Nomarski optics.

In later experiments, a horseradish peroxidase (HRP) conjugate of goat anti-mouse $\kappa$ light chain IgG was prepared using the heterobifunctional reagent, $N$-succinimidyl 3-(2-pyridyldithio)propionate (SPDP) (Carlsson et al., 1978). The conjugate was absorbed extensively with SCG homogenate to reduce background staining.

Electron microscopy. Because persistent background staining occurred in tissue from animals perfused with fixative, standard preparative procedures for electron microscopy were not used. Rats were overdosed with sodium pentobarbital and perfused with $0.12 \mathrm{M}$ Millonig's phosphate buffer at $37^{\circ} \mathrm{C}(\mathrm{pH} 7.3)$. Ganglia were removed rapidly and kept on ice whenever possible. Tissue was embedded in 6\% agar in Millonig's phosphate buffer. Fifty-micrometer sections along the long axis of the ganglion were cut using a Vibratome. After an additional 45min wash with ice cold buffer, the sections were fixed for 45 min at $4^{\circ} \mathrm{C}$ in $4 \%$ paraformaldehyde in Millonig's phosphate buffer. The total time from the sacrifice of the animal to fixation was no more than $3 \mathrm{hr}$.
Fixed sections were rinsed in buffer briefly, incubated for $30 \mathrm{~min}$ in $5 \%$ goat serum and then in hybridoma culture supernatants for $2 \mathrm{hr}$ at room temperature with gentle agitation. After washing, the sections were postfixed for $30 \mathrm{~min}$ in $0.1 \%$ glutaraldehyde, $4 \%$ paraformaldehyde in buffer. After a 2-hr wash, the sections were incubated in SCG-absorbed HRP conjugate for $1 \mathrm{hr}$ with shaking and then were incubated in DAB as described above except that development was carried out on ice.

Stained sections were processed and embedded for electron microscopy as described elsewhere (Matthew et al., 1981b). Dehydrated and infiltrated sections were flatembedded in Araldite between layers of Aclar plastic film (Allied Chemical) and then remounted on Beem capsules. Approximately $60-\mathrm{nm}$ thin sections were cut using a Sorvall MT-2 ultramicrotome. Grids were examined in a JEOL 100B electron microscope without heavy metal counterstaining.

\section{Results \\ Quantitation of Antigen Levels in Developing Ganglia}

Radioimmune assays were used to study the quantitative development of the synaptic vesicle and proteoglycan antigens during postnatal development, to determine whether both antigens increased with a similar time course, and to determine whether these changes paralleled those observed for neurotransmitter enzymes (cf., Black, 1978).

\section{Proteoglycan antibodies ( $P G 3$ and $P G$ 22)}

In Figure 1, $A$ and $B$ show the developmental curves for Ags associated with PG 3 and PG 22. The results are presented as fractions of adult activity, since only relative values are derived from the assay. Approximately $6 \%$ of the adult level of the determinant recognized by $\mathrm{PG} 3$ was present at birth (birth $=$ day 0 ). The level increased slowly until 1 week after birth, then increased rapidly, and reached a plateau that was equal to adult levels by 14 days. The difference between antigen levels at 3 and 7 days and that at 10 days was significant ( $l$ test; $p<$ $0.01)$. A similar curve was observed for PG 22 except that $15 \%$ of the adult level of antigen was present at birth, more than twice the fractional level of PG 3. PG 22 binding increased 6 -fold between days 10 and 14 , reaching the adult level at that time. The change in antigen levels from days 7 and 10 to that at 14 days was also significant ( $l$ lest; $p<0.01$ ).

\section{Vesicle antibody (SV 48)}

The use of SV 48 was preferred for binding assays because of its higher affinity, but SV 30 gave similar results. The Ag associated with SV 48 was present at less than $10 \%$ of the adult level at birth, increased rapidly between 10 and 14 days, remained stable for 1 week, and then gradually increased (Fig. $1 C$ ). The plateau level was only $50 \%$ of adult levels. However, the change from 3 to 14 days was significant ( $t$ test; $p<0.05$ ). It was not possible to determine the relative contributions of the 

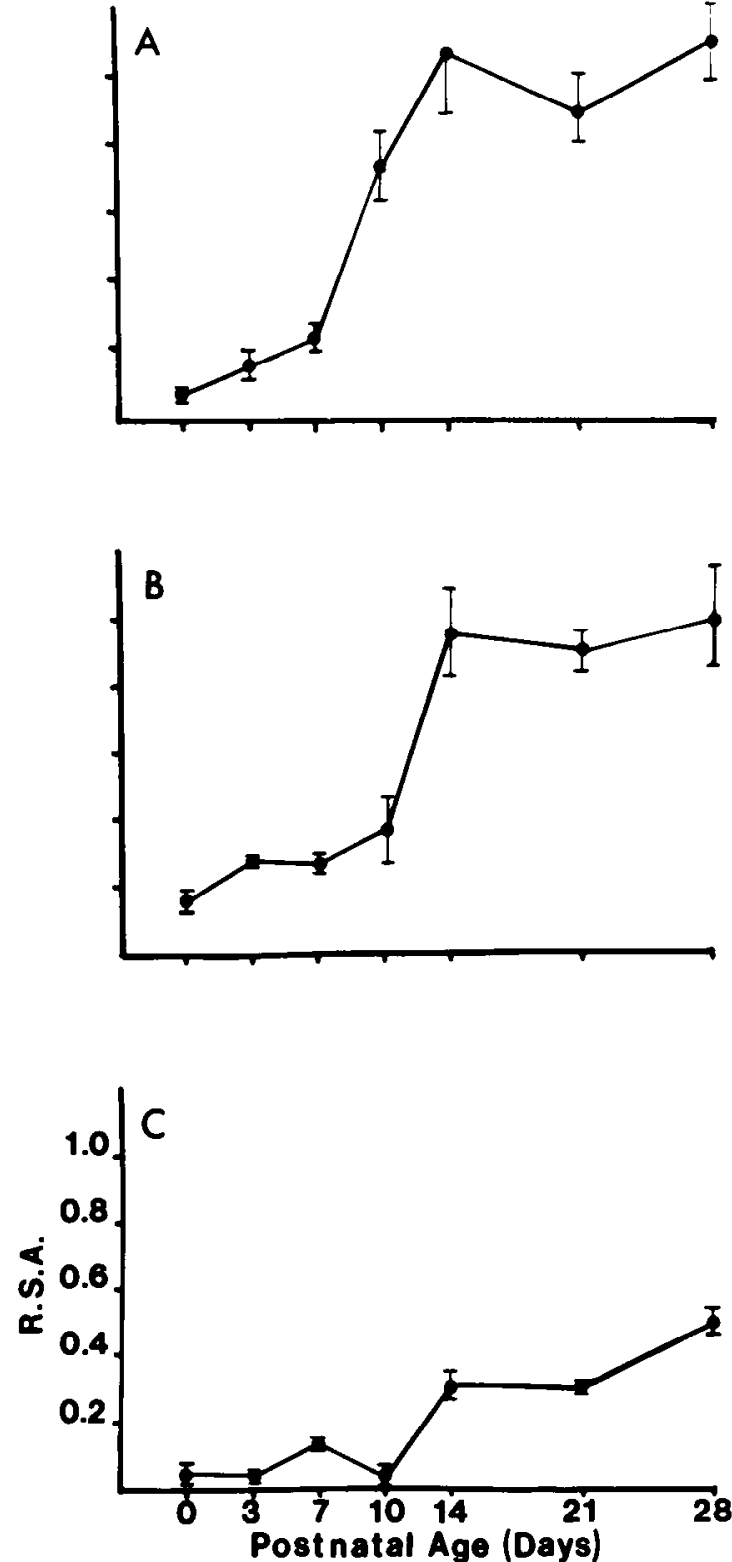

Figure 1. RIA quantitation of antigen levels in developing $\mathrm{SCG}$. The values were derived from $\mathrm{I}^{50}$ measurements as described under "Materials and Methods." The data are presented as fractions of adult levels (relative specific activity $(R . S . A)=$. $\mathrm{I}^{50}$ sample tissue $/ \mathrm{I}^{50}$ adult ganglion). The bars indicate $1 \mathrm{SE} . A$, PG 3; $B$, PG 22; $C$, SV 48. See the text for discussion.

accumulation of vesicles in presynaptic terminals and in cell cytoplasm of postsynaptic neurons.

\section{Assays of Other Tissues}

To investigate further the nature of the binding of these antibodies in peripheral ganglia, RIAs were carried out using homogenates of rat dorsal root ganglia (DRG), a sensory ganglion which contains few synapses. Matthew et al. (1981b) report heavy staining of synaptic terminals in the substantia gelatinosa of the rat thoracic spinal cord, the site of termination of DRG axons. This suggests that SV 30 and SV 48 recognize synaptic vesicles of sensory neurons. The results are summarized in Table
I. Buth proteoglycan Ags were present in DRG, but the vesicle antigen recognized by SV 48 was not detected $(<2 \%)$. These results are consistent with the evidence that PG 3 and PG 22 recognize a common molecule which is found on the surface of neurons and that SV 48 recognizes an antigen that is restricted to synaptic vesicles and therefore is present in very low levels in the cell bodies of DRG neurons.

\section{Immunocytochemistry of Antigens in SCG}

RIA gave information as to bulk changes in antigen levels during development. In order to study the distribution of $\mathrm{Ag}$ in the SCG and to determine whether changes in $\mathrm{Ag}$ distribution occurred during development, PAP staining of adult and developing SCG was carried out.

\section{Adult SCG}

Proteoglycan antibodies ( $P G 3$ and $P G$ 22). Light micrographs of PAP-stained sections of adult SCG using PG 3, PG 22, and a control (normal mouse serum) are shown in Figure 2. PG 3 showed patchy staining primarily between the principal neuronal cell bodies. There was no evidence for staining of non-neuronal cells based on this pattern, but it cannot be ruled out. An occasional cell with cytoplasmic staining was seen. PG 22 showed a broader staining pattern, heavy staining of the neuronal cell surface, patches between somas, and light but consistent staining of cell cytoplasm. No distinctive profiles of other cell types appeared to be stained. Control tissue incubated with normal mouse serum was virtually unstained.

Staining at the electron microscopic level by PG 3 is shown in Figure 3 . The plasma membrane of ganglionic neurons was stained heavily, both that of the cell body and of dendrites. The surface membrane of presynaptic profiles also was stained. There was no stain on membranes of non-neuronal cells. In addition, sometimes illdefined material in neuronal cell cytoplasm was stained. The stain was heavier toward the plasma membrane and was rare in the perinuclear region. Internal elements of dendritic and presynaptic profiles also were stained unevenly, with stain tending to be heavier toward the plasma membrane. This staining may reflect internal processing of the proteoglycan molecule (cf., Jourdian, 1979). There is also biochemical evidence for a large internal pool of this antigen (W. D. Matthew and L. F. Reichardt, manuscript in preparation).

Vesicle antibody ( $S V 30)$. The stained pattern of SV

TABLE I

Quantitation of antigen levels in dorsal root ganglion

The values were derived from two (PG 3 and PG 22) or four (SV 48) assays of whole tissue homogenate. The assays were performed as described under "Materials and Methods." See the text for discussion.

\begin{tabular}{llll}
\hline & PG 3 & PG 22 & SV 48 \\
\hline R.S.A. & 1.02 & 0.38 & N.D. \\
SE $^{c}$ & 0.13 & 0.005 & \\
\hline
\end{tabular}

${ }^{a}$ Relative specific activity $=\mathrm{I}^{5(0)}$ tissue $/ \mathrm{I}^{\mathrm{In})}$ adult $\mathrm{SCG}$.

${ }^{b}$ N.D., not detectable $(<0.02)$.

' $\mathrm{SE}=1$ standard error of the mean 

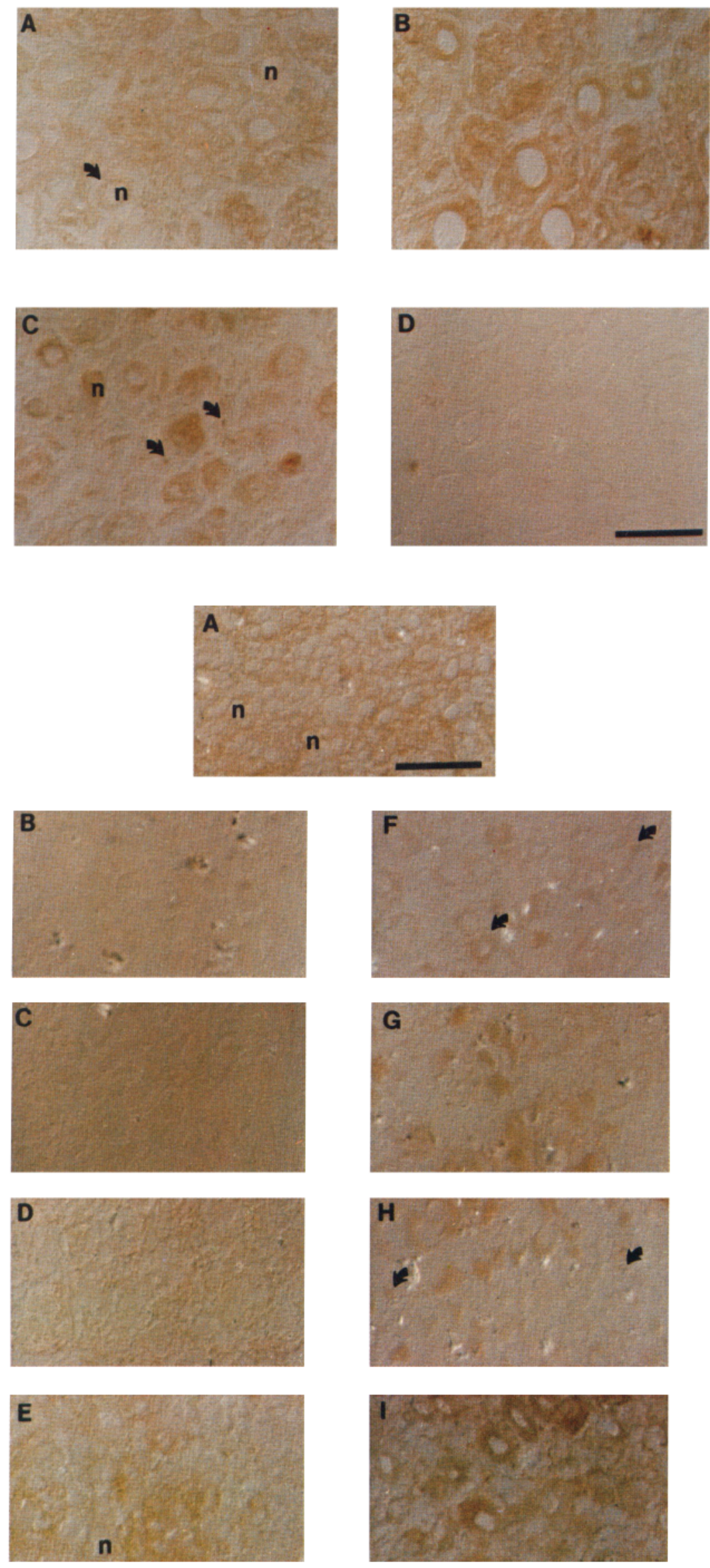

Figure 2. PAP staining patterns of $\mathrm{Abs}$ in adult rat SCG: PG 3, PG 22, and SV 30 with control (normal mouse serum in place of monoclonal $\mathrm{Ab}$ ). Six-micrometer frozen sections of unfixed adult rat SCG were incubated as described under "Materials and Methods." Nomarski optics; bar, $40 \mu \mathrm{m}$. $A$, Staining pattern using PG 3: note the dark staining between the principal ganglionic neurons $(n)$ with slight cytoplasmic stain (arrow). Prominent nuclei are unstained. $B$, Staining pattern of PG 22: heavy patchy staining between neurons with lighter staining of cell cytoplasm is evident. The nuclei are unstained. $C$, Staining pattern of SV 30: heavy staining of the principal neuron $(n)$ and cytoplasm is shown. The nuclei are unstained. Punctate staining of terminals between cell bodies (arrows) is also visible. This staining pattern is consistent with the known innervation of the SCG (cf., Gabella, 1976). $D$, Normal mouse serum control: serum was used at 1:10,000 in place of monoclonal $\mathrm{Ab}$. No staining is observed.

Figure 5. Ab staining of developing rat SCG. The tissue was prepared as in Figure 2. Bar, $40 \mu \mathrm{m}$. $A$, PG 22 PAP staining of neonatal (1-day-old) rat SCG. The section shows a patchy distribution of stain between cell bodies with light cytoplasmic and surface stain of neuronal cell bodies $(n)$. The neuron diameter is considerably smaller than in the adult. The staining pattern resembles that of the adult. $B$ to $E$, Staining patterns of PG 3. $B, 1$ day: no stain evident; $C, 7$ days: no stain; $D, 10$ days: faint suggestion of patchy stain reminiscent of adult pattern; $E, 14$ days: patchy stain visible around the cell bodies $(n)$. Like that of PG 22 , the PG 3 staining pattern resembles that of the adult. $F$ to $I$, Staining pattern of SV 30. F, 1 day: faint cytoplasmic stain (arrows); $G, 7$ days: increasing stain of cell cytoplasm with little evidence for punctate terminal stain; $H, 10$ days: first appearance of weak stain in regions between the cell bodies (arrows); $I, 14$ days: cytoplasmic and punctate terminal staining both evident. 

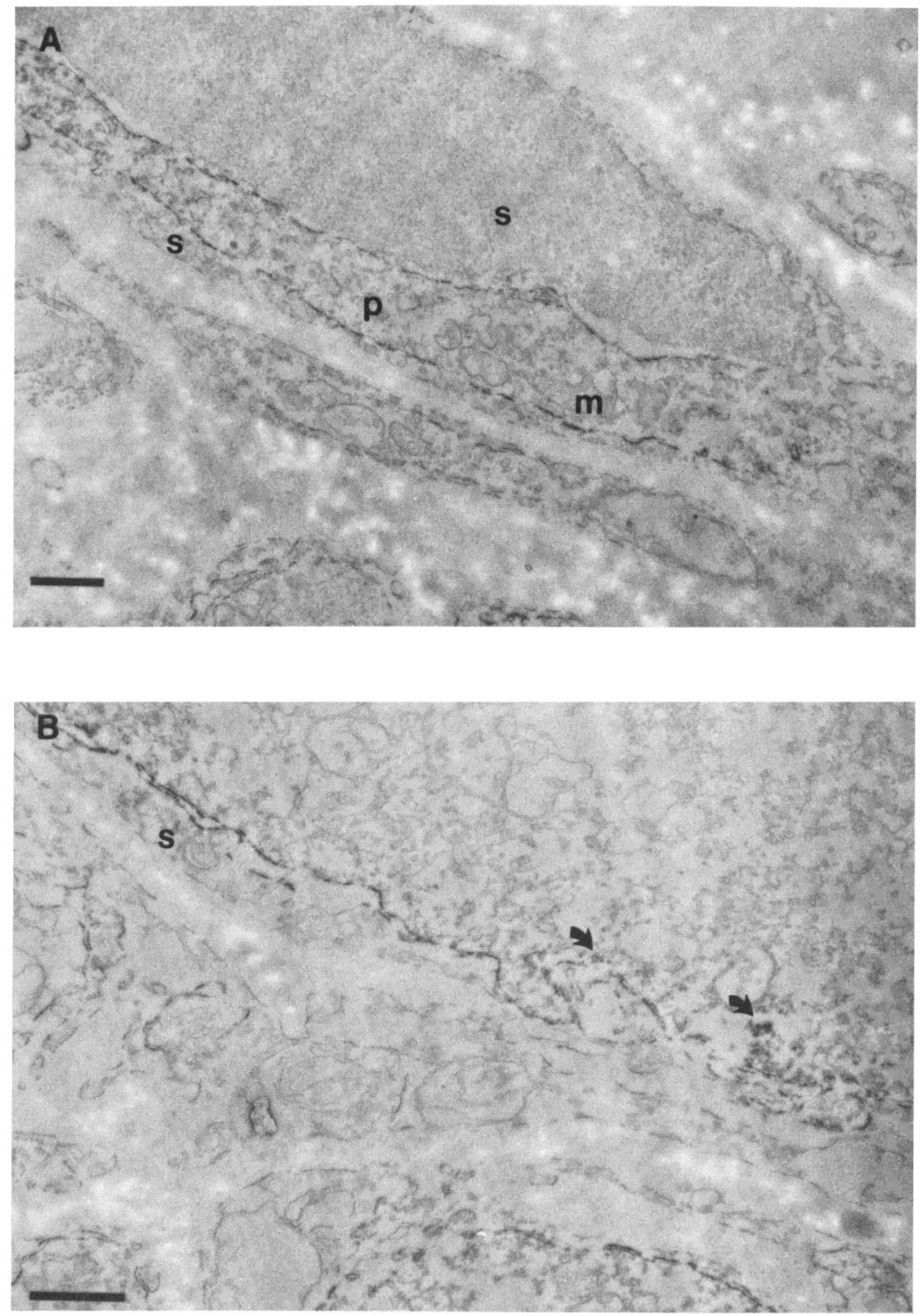

Figure 3. Electron microscopy of PG 3. HRP-goat anti-mouse conjugate was used as described under "Materials and Methods." Bars, $1.0 \mu \mathrm{m}$. A, Surface stain of the plasma membrane of a cell process $(p)$. Some irregular staining of the cytoplasm adjacent to membrane is evident. Mitochondria $(m)$ are unstained and no stain of the nucleus or cell process of the visible satellite cell $(s)$ is evident. $B$, Cell surface of the principal ganglionic neuron. The satellite cell process (arrows) is not stained. 

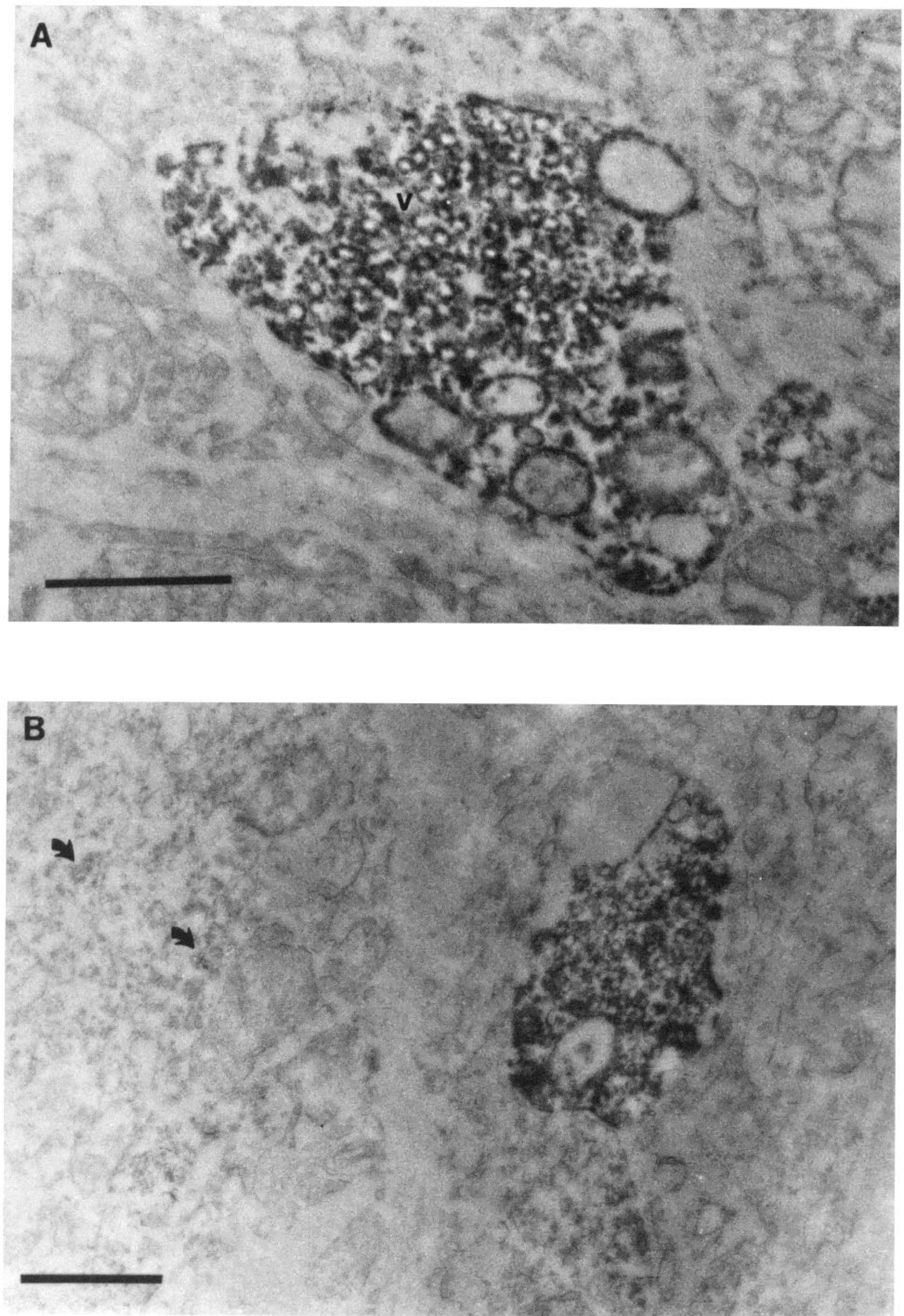

Figure 4. Ultrastructural staining pattern of SV 30 in adult rat SCG. Electron micrographs of HRP-IgG conjugate-stained tissue prepared as described under "Materials and Methods" and in Figure 3 are shown. Bars, $0.5 \mu \mathrm{m}$. A, Heavy staining of the outer surface of synaptic vesicles in terminal regions $(v)$. The adjacent mitochondrial membrane also is stained. In $B$, cytoplasmic stain is evident but not prominent (arrows). 
30 in adult SCG is shown in Figure $2 C$. SV 30 stained small punctate regions between ganglionic neurons and also prominently stained the cytoplasm of these cells. There was no staining of plasma membrane, cell nuclei, or other cell types. Initial screening indicated that SV 30 yielded better histology and, for this reason, was employed. SV 48 stained in the same pattern (not shown).

Electron micrographs of SV 30 staining (Fig. 4) showed heavily stained vesicles in presynaptic terminal profiles. The adjacent mitochondrial membrane also was stained. Because immunoprecipitation procedures specifically precipitated synaptic vesicles and not mitochondria (Matthew et al., 1981b), this stain was almost certainly the result of reaction product diffusion. Cytoplasmic staining, while dramatic at the light microscopic level, was not prominent at the ultrastructural level. The stain appeared diffusely distributed within the cell cytoplasm and did not appear to be associated with particular intracellular elements except for occasional vesicles.

\section{Developing SCG}

Immunocytochemistry of developing ganglia. PAP staining of developing ganglia was carried out to determine whether the localization of antigens changed during development. The results are summarized in Table II; the development of staining is shown in Figure 5.

Sections of ganglia from rats aged 1 to 28 days postnatal were examined. Of the three antibodies, only $\mathrm{PG}$ 22 stained neonatal tissue (Fig. 5A). The pattern of staining closely paralleled the adult pattern, although neuronal cell diameters were smaller in young animals. In Figure $5, B$ to $E$ show the development of staining using PG 3. Staining first appeared in sections from rats 10 days old. As with PG 22, the staining pattern resembled the adult pattern as soon as it became detectable. Staining intensified with further postnatal development.

The appearance of stain using SV 30 is shown in Figure $5, F$ to $I$. There was a suggestion of cytoplasmic staining in sections of ganglia from neonatal rats. SV 30 noticcably stained the cell cytoplasm of ganglia from 7-day-old rats. However, punctate staining did not become evident until 10 to 14 days after birth. It is not certain whether this result is due to the absence of antigen in terminals before this time or whether the antigen is below detectable levels in very young animals.

\section{TABLE II}

Appearance of antibody staining in developing rat SCG

The symbols used to indicate the degree of staining are: - , no stain above background; $+/-$, very faint stain; $+(++)$, positive stain graded for overall intensity. The values were derived from PAP-stained frozen sections. See the text and figures for details.

\begin{tabular}{lcccccc} 
& \multicolumn{6}{c}{ Postnatal Age (Days) } \\
\cline { 2 - 7 } Antibody & 1 & 7 & 10 & 14 & 21 & Adult \\
\hline PG 3 & - & - & $+/-$ & + & + & ++ \\
PG 22 & + & ++ & ++ & ++ & +++ & +++ \\
SV 30 & $+/-$ & $+^{\prime \prime}$ & + & $++b$ & ++ & ++ \\
Control & - & - & - & - & - & - \\
\hline
\end{tabular}

"Staining of cell cytoplasm only.

${ }^{b}$ Staining of cell cytoplasm and terminals.

"Normal mouse serum.

\section{Discussion}

The results of these experiments clearly indicate that it is possible to use monoclonal antibodies to monitor the developmental changes of their associated antigens. The levels of antigens associated with the neuronal cell surface and with synaptic vesicles increase significantly during the first few weeks after birth, with the most rapid changes in antigen levels occurring during the 2 nd postnatal week. This result closely parallels the time course of the postnatal increases in CAT and TH within the SCG (Black et al., 1971, 1972, 1979; Black, 1978). Antibodies bind to adult rat SCG in patterns consistent with the expected distribution of antigens. Within the limits of light microscopy, it appears that a major redistribution of these antigens does not occur during postnatal development, but electron microscopic evaluation of early staining patterns will be required to confirm this observation. This finding may be the result of the particular antigens under study; other antigens may resemble the acetylcholine receptor, which undergoes a redistribution during the formation of the neuromuscular junction (reviewed in Fambrough, 1979; Dennis, 1981). Our results provide further confirmation that the processes of synapse formation and neuronal maturation are closely linked.

Proteoglycan antibodies ( $P G 3$ and PG 22). Radioimmune assay indicates that the bulk levels of both antigenic determinants on the HeS proteoglycan increase during postnatal development. Immunocytochemical staining further indicates that this increase is associated specifically with the growth of ganglionic neurons and the expansion of their dendritic arborizations. Staining intensifies as the amount of neuronal plasma membrane increases. The increases in proteoglycan $\mathrm{Ag}$ levels do not simply reflect the general growth of the ganglion. In addition to increases in neuronal size and territory, marked glial proliferation and increases in connective tissue occur after birth. The increase in total ganglion protein is only 3-fold and is approximately linear throughout postnatal development (data not shown) as has been reported previously (Black et al., 1971). The increases in Ag level, as monitored by RIA, closely parallel the reported increases in 'TH levels associated with neuronal maturation (Black et al., 1971). A critical question which remains to be answered is whether the antigens studied in this report also would fail to increase normally in the absence of presynaptic input.

Significant changes in proteoglycan levels have been reported to occur postnatally in rat brain (Margolis et al., 1975; Jourdian, 1979) with the most rapid and striking period occurring between birth and 14 days. Margolis et al. (1975) reported that the concentrations of HeS and most other glycosaminoglycans decrease during postnatal development. However, the rate of synthesis of $\mathrm{HeS}$ proteoglycans increased 4 -fold, with the majority of the increase observed after the 1st week of life. Metabolism of other glycosaminoglycans in brain did not change appreciably. They proposed that this increase in HeS proteoglycan metabolism reflects the possible role of the proteoglycan in maturation processes which occur postnatally at terminals, including the binding, storage, and 
release of amine neurotransmitters. This proposal is compatible with our observations.

There is some evidence for developmental changes in the processing of the HeS proteoglycan recognized by PG 3 and PG 22. We have observed that, at birth, there is comparatively less carbohydrate $\mathrm{Ag}$ (PG 3 ) than core protein-associated Ag (PG 22). PG 3 did not stain the SCG until 10 to 14 days after birth, while PG 22 stained the SCG at birth. These results suggest that the processing of this $\mathrm{HeS}$ proteoglycan may vary during development; PG 3 recognition of its antigenic determinant may be delayed until the side chains of the proteoglycan resemble those in the adult. Further experiments are required to confirm this possibility.

There is evidence that cell surface glycoproteins play roles in cell-cell recognition and adhesion during the development of neural systems (see Gottlieb and Glaser, 1980 , for review) and that rapid changes in cell adhesiveness can occur during development. Lander et al. (1982) reported the isolation of a HeS proteoglycan from bovine endodermal conditioned medium which promotes neurite outgrowth in culture. The antibodies PG 3 and PG 22 immunoprecipitate a similar neurite outgrowth factor produced by some rat cells, including the neuronal cell line, PC12. A variant of PC12, lacking the surface $\mathrm{HeS}$ proteoglycan, also does not synthesize the neurite outgrowth factor (A. D. Lander, R. Greenspan, W. D. Matthew, and L. F. Reichardt, unpublished observations). Therefore, the HeS proteoglycan defined by PG 3 and PG 22 seems likely to participate in cell-cell interactions and axon and dendrite growth in vivo. If so, it is not surprising that increased levels of this proteoglycan appear in the SCG at the time of presynaptic axon invasion and postsynaptic dendritic elaboration. Further experiments, though, are needed to test in vivo for these possible functions.

Vesicle antibodies ( $S V 30$ and $S V$ 48). Radioimmune assays using SV 48 indicate that postnatal increases in antigen levels parallel previous assessments of synaptogenesis and neuronal maturation in the SCG (Eranko, 1972; Black et al., 1979). These experiments do not show what fraction of the antigen increase is the result of the accumulation of vesicles at newly formed presynaptic terminals and what fraction results from increases in the number of vesicles within the cell body. However, SCG immunocytochemistry reveals that the vesicle-associated protein can be visualized in neuronal cell cytoplasm shortly after birth. It is known that the ganglionic cell bodies accumulate adrenergic vesicles during development (Eranko, 1972) and that neurons contain significant numbers of vesicles in the adult. However, at the ultrastructural level, the staining within the cell cytoplasm was not dramatic. It should be noted that the rat SCG is the only neuronal tissue in which staining other than that at terminals has been observed (cf., Matthew et al., 1981a, b).

Punctate immunocytochemical staining of presumed presynaptic terminals does not appear until days 10 to 14. Smolen and Raisman (1980) reported that, as assessed by electron microscopy, the period of the most rapid formation of synapses occurs during the 1st postnatal week. This increase precedes the increase in CAT levels reported earlier (Black el al., 1971, 1972; Black and Geen, 1973). However, the synapses formed during the first few days after birth were morphologically immature. Our results support Smolen and Raisman's (1980) statement that the maturation of synapses formed immediately postnatally continues during the 2 nd week of life.

As with the neuronal cell surface proteoglycan, we would like to determine whether the accumulation of the synaptic vesicle-associated $\mathrm{Ag}$ in cell bodies is affected by trans-synaptic influences. A possible approach to this question is to denervate both adult and developing ganglia and to measure the survival of antigen within the cell body.

By combining developmental studies with attempts to perturb normal development using antibodies, it may be possible to elucidate at least some of the interactions occurring between molecules which result in the development of a functioning neural system. Such research, carried out both in vitro and in vivo, should permit the analysis of factors influencing development to a degree not possible using more traditional methods.

\section{References}

Black, I. B. (1978) Regulation of autonomic development. Annı. Rev. Neurosci. 1: 183-211.

Black, I. B., and S. C. Geen (1973) Transsynaptic regulation of adrenergic neuron development: Inhibition by ganglionic blockade. Brain Res. 63: 291-302.

Black, I. B., I. A. Hendry, and L. L. Iversen (1971) Transsynaptic regulation of growth and development of adrenergic neurons in a mouse sympathetic ganglion. Brain Res. 34: 229240.

Black, I. B., I. A. Hendry, and L. L. Iversen (1972) Effects of surgical decentralization and nerve growth factor on the maturation of adrenergic neurons in a mouse sympathetic ganglion. J. Neurochem. 19: 1367-1377.

Black, I. B., M. D. Coughlin, and P. Cochard (1979) Factors regulating neuronal differentiation. In Society for Neuroscience Symposia. Vol. 4: Aspects of Developmental Neurobiology, pp. 184-187, Society for Neuroscience, Bethesda, MD.

Carlsson, J., H. Drevin, and R. Axen (1978) Protein thionation and reversible protein-protein conjugation. Biochem. J. 173: 723-737.

Coughlin, M. D., M. D. Dibner, D. M. Boyer, and I. B. Black (1978) Factors regulating development of an embryonic mouse sympathetic ganglion. Dev. Biol. 66: 513-528.

Dennis, M. J. (1981) Development of the neuromuscular junction: Inductive interactions between cells. Annu. Rev. Neurosci. 4: 43-68.

Eranko, L. (1972) Ultrastructure of the developing sympathetic nerve cell and the storage of catecholamines. Brain Res. 46: 159-175.

Eranko, O., and L. Eranko (1971) Small, intensely fluorescent granule-containing cells in the sympathetic ganglion of the rat. Prog. Brain Res. 31: 39-51.

Fambrough, D. M. (1979) Control of acetylcholine receptors in skeletal muscle. Physiol. Rev. 59: 165-227.

Gabella, G. (1976) Structure of the Autonomic Nervous System, Chapman and Hall, London.

Gottlieb, D. I., and L. Glaser (1980) Cellular recognition during neuronal development. Annu. Rev. Neurosci. 3: 303-318.

Greif, K. F., W. D. Matthew, and L. F. Reichardt (1981) Postnatal development of rat superior cervical ganglion as monitored by monoclonal antibodies. Soc. Neurosci. Abstr. 7: 669 .

Hendry, I. A., and C. E. Hill (1980) Denervation-induced de- 
creases in enzyme activity of rat superior cervical ganglia differ in vivo and in vitro. Brain Res. 200: 201-205.

Jones, D. H., and A. I. Matus (1974) Isolation of synaptic plasma membranes from brain by combined flotation-sedimentation density gradient centrifugation. Biochim. Biophys. Acta 356: 276-287.

Jourdian, G. W. (1979) Biosynthesis of glycosaminoglycans. In Complex Carbohydrates of Nervous Tissue, R. U. Margolis and R. K. Margolis, eds, pp. 103-125, Plenum Press, New York.

Klinman, N. R. (1972) The mechanism of antigenic stimulation of primary and secondary clonal precursor cells. J. Exp. Med. 136: 241-260.

Kondo, H., N. J. Duncan, and G. D. Pappas (1980) A light and electron microscopic study of the rat superior cervical ganglion cells by intra-cellular horseradish peroxidase labelling. Brain Res. 197: 193-199.

Lander, A. D., D. K. Fujii, D. Gospodarowicz, and L. F. Reichardt (1982) Characterization of a factor that promotes neurite outgrowth: Evidence linking activity to a heparan sulfate proteoglycan. J. Cell Biol., in press.

Margolis, R. U., R. K. Margolis, L. B. Chang, and C. Preti (1975) Glycosaminoglycans of brain during development. Biochemistry 14: 85-88.

Matthew, W. D., L. F. Reichardt, and L. Tsavaler (1981a) Monoclonal antibodies to synaptic membranes and vesicles. In Monoclonal Antibodies to Neural Antigens, R. McKay, M. Raff, and L. Reichardt, eds., pp. 167-180, Cold Spring Harbor Laboratory, Cold Spring Harbor, NY.

Matthew, W. D., L. Tsavaler, and L. F. Reichardt (1981b) Identification of a synaptic vesicle specific membrane protein with a wide distribution in neuronal and neurosecretory tissue. J. Cell Biol. 91: 257-269.

Smolen, A., and G. Raisman (1980) Synapse formation in the rat superior cervical ganglion during normal development and after neonatal deafferentation. Brain Res. 181: 315-323.

Sternberger, L. (1979) Immunocytochemistry, John Wiley and Sons, New York. 